\title{
Increased Rate of Formation of Small-Sized Platelet Aggregates in Patients With Acute Coronary Syndromes
}

Shinzo Miyamoto, MD; Hisao Ogawa, MD; Hirofumi Soejima, MD;

Keiji Takazoe, MD; Ichiro Kajiwara, MD; Tomohiro Sakamoto, MD; Michihiro Yoshimura, MD; Kiyotaka Kugiyama, MD; Hirofumi Yasue, MD

\begin{abstract}
Coronary thrombosis has been implicated in the pathogenesis of acute coronary syndromes, and platelet activation plays a pivotal role in the pathogenesis of coronary thrombus. A new platelet aggregometer using a laserlight scattering beam was trialled for assessment of platelet aggregation. Platelet aggregability, especially smallsized platelet aggregates, was investigated on admission using the laser-light scattering method and again after treatment in 23 patients with acute coronary syndromes. The platelet aggregability in 14 patients with stable exertional angina and in 14 control subjects was also examined. On admission, the number of small- and medium-sized platelet aggregates in the acute coronary syndromes group was significantly greater than in the stable exertional angina group or control group. However, the number of large-sized platelet aggregates on admission was not increased in the acute coronary syndromes group. Furthermore, the number of small- and medium-sized platelet aggregates decreased significantly after treatment in the acute coronary syndromes group. The increased number of small-sized platelet aggregates may sensitively reflect attacks of thrombosis in patients suffering acute coronary syndromes. (Jpn Circ J 2000; 64: 647-652)
\end{abstract}

Key Words: Acute coronary syndromes; Laser-light scattering method; Platelet aggregability

C oronary thrombosis plays an important role in the pathogenesis of acute coronary syndromes, including unstable angina, acute myocardial infarction and ischemic sudden death. ${ }^{1-3}$ We and other investigators ${ }^{4-8}$ have reported that the plasma level of fibrinopeptide $\mathrm{A}$, a sensitive marker of thrombin generation, and the activity of plasma plasminogen activator inhibitor, 9,10 an indicator of the impairment of fibrinolysis, increase in patients with unstable angina and acute myocardial infarction!!

Platelet activation therefore plays an important role in the pathogenesis of acute coronary syndromes! ${ }^{2-14}$ Platelet aggregation, conventionally measured using either the optical density method ${ }^{15}$ or impedance method, ${ }^{16}$ is indispensable for the clinical evaluation of platelet function. A recently developed platelet aggregability measuring system using a laser-light scattering method 17,18 is capable of monitoring the increase in the size of small aggregates ( 9 $25 \mu \mathrm{m}$, ie, $\leq 100$ platelets), which can not be detected with the conventional methods. It has been demonstrated that the formation of medium- and large-sized platelet aggregates begins with small-sized platelet aggregates, ${ }^{17,18}$ so the production of small-sized platelet aggregates is very important in initial thrombus formation. Using the new aggregometer, Eto et al have shown that the number of small aggregates increased in acute coronary syndromes 19

In the present study, also using the new laser-light scatter-

(Received February 25, 2000; revised manuscript received June 7, 2000; accepted June 9, 2000)

Department of Cardiovascular Medicine, Kumamoto University School of Medicine, Kumamoto, Japan

Mailing address: Hisao Ogawa, MD, Department of Cardiovascular Medicine, Kumamoto University School of Medicine, 1-1-1 Honjo, Kumamoto 860-8556, Japan. E-mail: ogawah@kumamoto-u.ac.jp ing method, we measured platelet aggregability, both on admission and after treatment, in patients with acute coronary syndromes (acute myocardial infarction and unstable angina pectoris) and those with stable exertional angina, as well as control subjects. We compared the results with data from the conventional optical density method.

\section{Methods}

\section{Study Population}

Fifty-one patients (35 men, 16 women; mean age, $65 \pm 1$ years, mean \pm SEM, range $40-84$ ), who underwent diagnostic cardiac catheterization, were studied. They were divided into an acute coronary syndromes group, a stable exertional angina group, and a control group. The acute coronary syndromes group consisted of 23 patients (10 acute myocardial infarction and 13 unstable angina pectoris; 16 men, 7 women; mean age $66 \pm 2$ years) who had attacks of chest pain associated with ST segment elevation or depression on ECG. The 10 patients with acute myocardial infarction ( 7 men, 3 women) were admitted within $12 \mathrm{~h}$ of symptom onset and the diagnosis was made on the basis of chest pain persisting for $\geq 30 \mathrm{~min}$, ST-segment elevation of $>0.2 \mathrm{mV}$ in more than 2 contiguous leads on a standard 12-lead ECG, and elevation of the serum creatine kinase-MB isoenzyme level to more than twice the upper limit of normal. The average time from the onset of symptoms to admission was $7.4 \pm 1.8$ (mean \pm SEM) h (range $1-12$ ). The 13 patients with unstable angina pectoris comprised 9 men and 4 women and their condition was defined as a crescendo pattern of chest pain at rest with documented transient ST-segment depression or ST-segment elevation of at least $0.1 \mathrm{mV}$ in at least 2 contiguous ECG leads. Their last spontaneous attack 
was required to have occurred within $1 \mathrm{~h}$ of entry into the study. There were no patients with ST-segment changes lasting more than $30 \mathrm{~min}$, with new $\mathrm{Q}$ waves, or with raised plasma concentrations of creatine kinase or creatine kinaseMB. The stable exertional angina group consisted of 14 patients ( 11 men, 3 women; mean age $68 \pm 2$ years) who had typical exertional angina and $\geq 90 \%$ narrowing of the major coronary artery. The most recent attack in each patient of this group had occurred at least 4 weeks before entry into the study. No changes in the frequency of angina, precipitating factors, or responses to sublingual nitroglycerin were noted in the previous 3 months in this study group. The control group consisted of 14 patients ( 8 men, 6 women; mean age $59 \pm 2$ years) who had atypical chest pain unaccompanied by ECG changes and without significant coronary arterial stenosis ( $\leq 25 \%$ of luminal diameter), or coronary spasm, as demonstrated by an intracoronary injection of acetylcholine.

The stable exertional angina and control groups were selected from patients undergoing elective cardiac catheterization during the same period as the acute coronary syndromes group and were matched for age, gender and other clinical variables.

In the patients with acute coronary syndromes, oral medications were usually started on the first day of admission and were not discontinued at the time of coronary arteriography. In the patients with stable exertional angina, oral medications were usually administrated in hospital. The study patients were not treated with antiplatelet drugs other than aspirin. Factors suspected of reflecting platelet aggregation, such as the hematocrit, platelet count and total cholesterol, were not significantly different between the 3 groups (Table 1). Patients taking coumarin anticoagulant were excluded from this study. The study protocol was approved by the Ethics Committee and written informed consent was obtained from each patient and his or her family.

\section{Coronary Arteriography}

Emergency coronary arteriography was performed on admission in 6 patients with acute myocardial infarction. In 4 patients with acute myocardial infarction and 13 with unstable angina pectoris, medical treatment were prescribed and coronary arteriography was performed $2-4$ weeks after admission. In the patients with stable exertional angina and the control subjects, coronary arteriography was performed by the Judkins technique using contrast material in the morning when the patients were fasting.

After heart rate and blood pressure measurements, control angiography of the left main and right coronary arteries was performed. The patients with acute coronary syndromes who did not have significant organic stenosis and the control subjects received an intracoronary acetylcholine injection to provoke coronary spasm $2^{20}$ Thereafter, isosorbide dinitrate was administered to detect organic coronary lesions.

\section{Blood Sampling}

Blood samples for measuring the platelet count, hematocrit and lipid concentration were drawn using a 21 -gauge needle inserted into an antecubital vein while the subject lay recumbent; $4.5 \mathrm{ml}$ of blood was collected into tubes containing sodium citrate. Venous blood samples were obtained on admission before the start of heparin or other drugs in patients with acute coronary syndromes. Because it is considered that platelet aggregability increases signifi- cantly during the morning, ${ }^{21}$ repeat venous blood samples from the 23 acute coronary syndrome patients were taken at $07.00 \mathrm{~h}$ in the fasting state $2-4$ weeks after admission. Blood samples from the stable exertional angina and control subjects were obtained at $07.00 \mathrm{~h}$ in the fasting state after admission. The sample was left for $15 \mathrm{~min}$ at room temperature, then centrifuged at $150 \mathrm{G}$ for $10 \mathrm{~min}$ at room temperature in order to obtain platelet-rich plasma. The remaining sample was then centrifuged at $300 \mathrm{G}$ for $10 \mathrm{~min}$ at room temperature in order to obtain platelet-poor plasma.

\section{Platelet Aggregometer}

Platelet aggregability was assessed using the aggregometer (PA-200, Kowa, Tokyo, Japan) recently developed by Ozaki et al, ${ }^{17,18}$ the principles of which have been described previously. Briefly, a laser beam measuring $40 \mu \mathrm{m}$ in diameter was generated using a $20 \mathrm{~mW}$ diode laser $(675 \mathrm{~nm}$, Toshiba, Tokyo, Japan) and passed through platelet-poor plasma $(300 \mu \mathrm{l})$ stirred in a $5-\mathrm{mm}$ internal diameter cylindrical glass cuvett. The light scattered from the observation volume $(48 \times 140 \times 20 \mu \mathrm{m})$ was detected by a photocell array, with light intensity corresponding to particle size. The signal frequency was recorded at 10 -s intervals. Data were expressed as the change over time (s) in the number of aggregates (counts/s) of individual sizes (determined by light intensity, expressed in volts). Particles with an intensity of $25-400 \mathrm{mV}$ represented small-sized platelet aggregates $(9-25 \mu \mathrm{m})$, those with an intensity of $400-1,000 \mathrm{mV}$ represented medium-sized platelet aggregates $(25-50 \mu \mathrm{m})$, and those with an intensity of $1,000-2,048 \mathrm{mV}$ represented large-sized platelet aggregates $(50-70 \mu \mathrm{m})$. Changes in total intensity were recorded at 10 -s intervals for $5 \mathrm{~min}$. Platelet aggregation was assessed in a spontaneous state and in the presence of $\operatorname{ADP}(0.5,1.0$, and $3.0 \mu \mathrm{mol} / \mathrm{L} ; 1.0 \mu \mathrm{mol} / \mathrm{L}$ of ADP was used as the aggregating reagent because a preliminary study had determined that detection of smallsized platelet aggregation was most sensitive at this concentration). We compared the results with data from the conventional optical density method.

\section{Effect of Aspirin}

Aspirin therapy is of proven value in the treatment of acute myocardial infarction as well as long-term in patients with a wide range of prior manifestations of cardiovascular disease.22 To examine whether aspirin had an influence on platelet aggregation, we measured platelet aggregates before and after $81 \mathrm{mg} /$ day of aspirin treatment in 12 patients with stable exertional angina as a preliminary study.

\section{Statistical Analysis}

All data are expressed as mean \pm SEM. The comparisons of continuous data among the 3 groups (acute coronary syndromes group, stable exertional angina group, and control group) were analyzed by a one-way analysis of variance. When these results were statistically significant, they were analyzed by Fisher's test. Changes in platelet aggregation in patients with acute coronary syndromes on admission and after treatment were analyzed by paired $t$ test. The frequency data were compared by a chi-square test. Probability levels $<0.05$ were considered significant.

\section{Results}

Patients' Characteristics and Clinical Outcomes

The clinical characteristics of the study population are 
Table 1 Clinical Characteristics of the Study Groups

\begin{tabular}{|c|c|c|c|}
\hline Characteristics & $\begin{array}{l}\text { Acute coronary } \\
\text { syndrome }(n=23)\end{array}$ & $\begin{array}{c}\text { Stable exertional } \\
\text { angina }(n=14)\end{array}$ & $\begin{array}{c}\text { Controls } \\
(n=14)\end{array}$ \\
\hline \multicolumn{4}{|l|}{ Age (years) } \\
\hline Mean & $66 \pm 2$ & $68 \pm 2$ & $59 \pm 3$ \\
\hline Range & $40-84$ & $50-77$ & $46-81$ \\
\hline Gender (n) $M / F$ & $16 / 7$ & $11 / 3$ & $8 / 6$ \\
\hline Hematocrit (\%) & $36.5 \pm 1.3$ & $36.7 \pm 1.0$ & $38.0 \pm 1.6$ \\
\hline Platelet count $\left(\times 10^{4} / \mathrm{mm}\right)$ & $19.3 \pm 1.1$ & $19.6 \pm 2.0$ & $18.5 \pm 1.7$ \\
\hline \multicolumn{4}{|l|}{ Coronary risk factors } \\
\hline Hypertension $(B P \geq 140 / 90 \mathrm{mmHg}, n)$ & 16 & 8 & 9 \\
\hline Diabetes mellitus $(n)$ & 13 & 5 & 2 \\
\hline Obesity $(B M I \geq 25, n)$ & 7 & 4 & 5 \\
\hline Smoking $(n)$ & 14 & 8 & 3 \\
\hline \multicolumn{4}{|l|}{ Hyperlipidemia $(\mathrm{mg} / \mathrm{dl})$} \\
\hline Serum total-cholesterol & $173 \pm 6$ & $179 \pm 13$ & $193 \pm 14$ \\
\hline Serum $L D L$-cholesterol & $99 \pm 7$ & $106 \pm 11$ & $123 \pm 10$ \\
\hline Serum HDL-cholesterol & $43 \pm 3$ & $49 \pm 6$ & $56 \pm 4$ \\
\hline Serum triglyceride & $131 \pm 39$ & $119 \pm 22$ & $138 \pm 19$ \\
\hline \multicolumn{4}{|l|}{$\begin{array}{c}\text { Extent of coronary vessel organic stenosis } \\
\geq 75 \% \text { stenosis }(n)\end{array}$} \\
\hline O-vessel & 3 & 0 & 14 \\
\hline 1-vessel & 10 & 9 & 0 \\
\hline 2-vessel & 3 & 2 & 0 \\
\hline 3-vessel & 7 & 3 & 0 \\
\hline \multicolumn{4}{|l|}{ Drug } \\
\hline Aspirin & 7 & 10 & 0 \\
\hline Calcium antagonists & 6 & 10 & 0 \\
\hline Long-acting nitrates & 5 & 6 & 0 \\
\hline$\beta$-blockers & 0 & 6 & 0 \\
\hline Angiotensin-converting enzyme inhibitors & 2 & 4 & 0 \\
\hline
\end{tabular}

Values are mean $\pm S E M$. BMI, body mass index; $B P$, blood pressure.

Table 2 Treatment of Patients With Acute Coronary Syndromes

\begin{tabular}{|c|c|c|c|c|}
\hline $\begin{array}{l}\text { Age (years) } \\
\text { /Gender }\end{array}$ & Diagnosis & Treatment & Culprit lesion & Medication before admission \\
\hline $63 / M$ & $A M I$ & Direct PTCA & $R C A$ & None \\
\hline $80 / F$ & $A M I$ & Direct PTCA & $L C x$ & None \\
\hline $46 / F$ & $A M I$ & Direct PTCA & $L A D$ & None \\
\hline $79 / F$ & $A M I$ & Direct PTCA & $L A D$ & None \\
\hline $72 / M$ & $A M I$ & Direct PTCA & $L A D$ & None \\
\hline $40 / M$ & $A M I$ & $I C T+$ rescue $P T C A$ & $R C A$ & None \\
\hline $84 / M$ & $A M I$ & Medication & $L A D$ & None \\
\hline $70 / M$ & $A M I$ & Medication & $L A D$ & None \\
\hline $83 / M$ & $A M I$ & Medication & $L C x$ & None \\
\hline $70 / M$ & $A M I$ & Medication & $L C x$ & None \\
\hline $69 / M$ & UAP & Medication & $L C x$ & None \\
\hline $52 / M$ & $U A P$ & Medication & $R C A$ & Aspirin, calcium antagonist, nitrate \\
\hline $72 / M$ & $U A P$ & Medication & $L A D$ (Coronary spasm) & Calcium antagonist \\
\hline $57 / F$ & UAP & Medication & LAD (Coronary spasm) & Calcium antagonist \\
\hline $57 / M$ & UAP & Medication & $L C x$ & Aspirin, nitrate \\
\hline $69 / M$ & $U A P$ & Medication & $L C x($ Coronary spasm $)$ & None \\
\hline $51 / M$ & $U A P$ & Medication & $L A D$ & Aspirin, nitrate, $A C E$ inhibitor \\
\hline $64 / M$ & $U A P$ & Medication & $L C x$ & Aspirin, calcium antagonist, nitrate \\
\hline $73 / F$ & $U A P$ & Medication & $L A D$ & None \\
\hline $71 / F$ & $U A P$ & Medication & $L A D$ & Aspirin, calcium antagonist \\
\hline $52 / M$ & UAP & Medication & $L C x$ & Aspirin, calcium antagonist \\
\hline $71 / F$ & UAP & Medication & $L A D$ & None \\
\hline $68 / M$ & UAP & Medication & $L A D$ & Aspirin, nitrate, $A C E$ inhibitor \\
\hline
\end{tabular}

PTCA, percutaneous transluminal coronary angioplasty; ICT, intracoronary thrombolysis; AMI, acute myocardial infarction; UAP unstable angina pectoris; $R C A$, right coronary artery; $L A D$, left anterior descending artery; $L C x$, left circumflex artery; ACE, angiontensin-converting enzyme.

detailed in Table 1. There were no significant differences among the 3 groups in terms of age, sex, hypertension, smoking, diabetes mellitus, obesity, serum total cholesterol, serum high density lipoprotein (HDL) cholesterol, serum low density lipoprotein (LDL) cholesterol or serum triglyc- eride levels. The medications of the patients with acute coronary syndromes are detailed in Table 2 . Of the patients with acute myocardial infarction, 6 of 10 had emergency coronary angiography, and 5 of 6 patients underwent direct percutaneous transluminal coronary angioplasty (PTCA) (3 
A

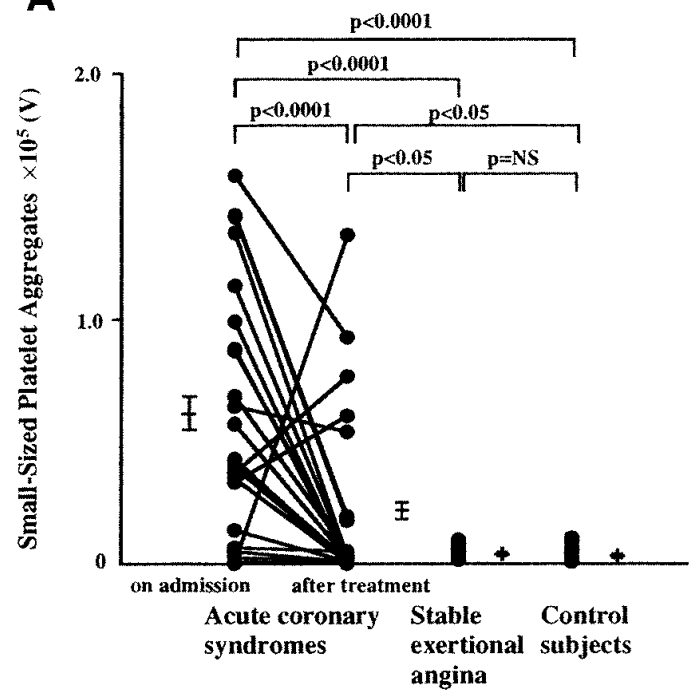

C

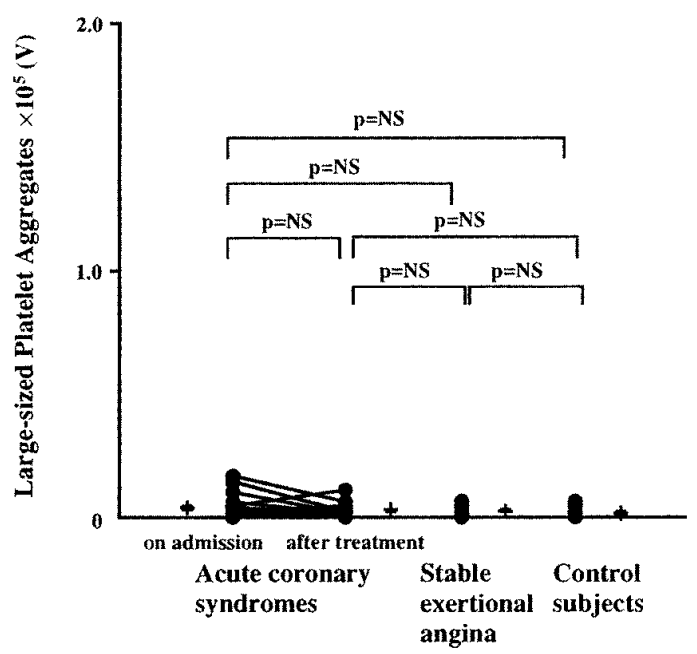

$\mathbf{B}$

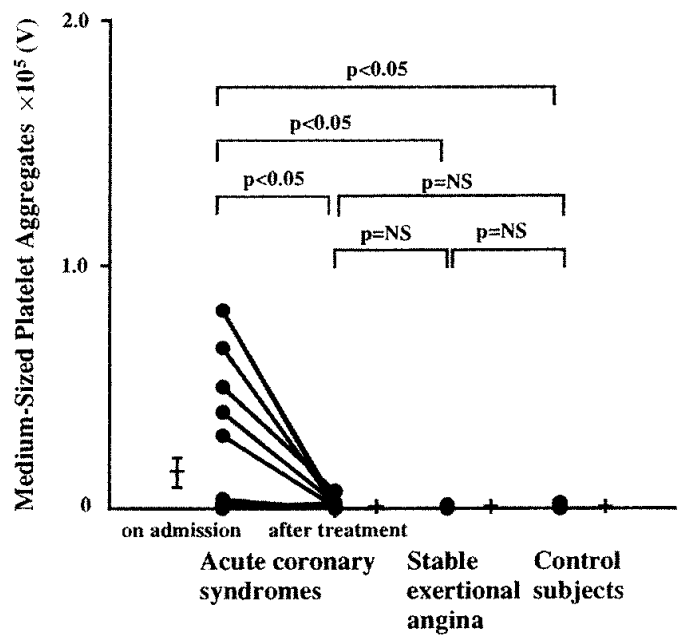

D

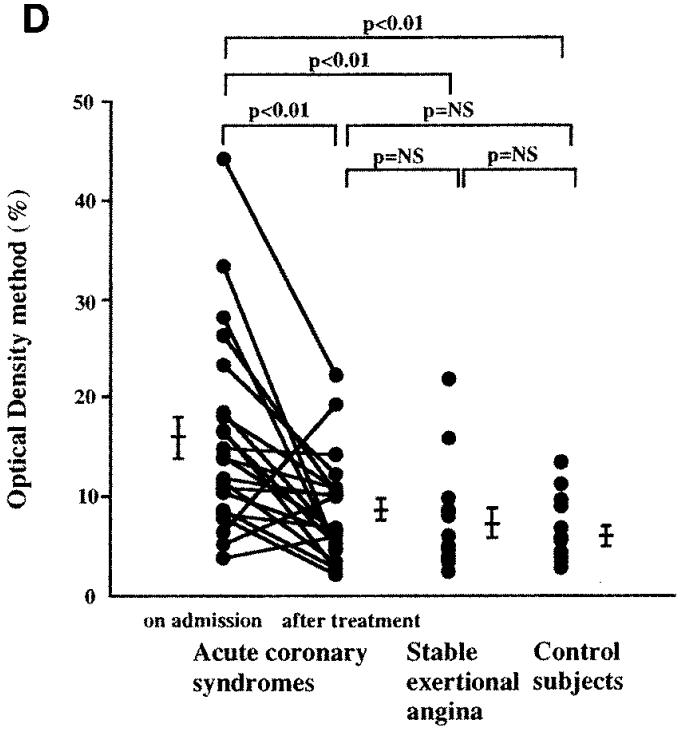

Fig 1. Comparison of the number of platelet aggregates using the laser-light scattering method and the optical density method. (A) Smallsized platelet aggregates, (B) medium-sized platelet aggregates, (C) large-sized platelet aggregates, (D) optical density method.

Table 3 Comparison Among the 3 Groups

\begin{tabular}{|c|c|c|c|c|}
\hline \multirow{2}{*}{ Platelet aggregates } & \multicolumn{2}{|c|}{ Acute coronary syndromes } & \multirow{2}{*}{$\begin{array}{c}\text { Stable exertional } \\
\text { angina }\end{array}$} & \multirow{2}{*}{ Controls } \\
\hline & On admission & After treatment & & \\
\hline Small-sized $(V)$ & $6.1 \pm 1.1 \times 10^{4 * * * h t}$ & $2.2 \pm 0.8 \times 10^{4 *}$ & $3.5 \pm 0.8 \times 10^{3}$ & $2.6 \pm 0.8 \times 10^{3}$ \\
\hline Medium-sized (V) & $1.5 \pm 0.6 \times 10^{4 * * * i}$ & $1.4 \pm 0.4 \times 10^{3}$ & $8.6 \pm 1.0 \times 10^{2}$ & $1.0 \pm 0.2 \times 10^{3}$ \\
\hline Large-sized (V) & $3.8 \pm 0.9 \times 10^{3}$ & $2.9 \pm 0.5 \times 10^{3}$ & $2.7 \pm 0.5 \times 10^{3}$ & $1.6 \pm 0.5 \times 10^{3}$ \\
\hline Optical density method (\%) & $16.0 \pm 2.1^{*+\dagger}$ & $8.7 \pm 1.1$ & $7.5 \pm 1.5$ & $6.3 \pm 1.0$ \\
\hline
\end{tabular}

Values are mean $\pm S E M$.

$* * * p<0.0001$ vs stable exertional angina and control subjects, $* * p<0.01$ vs stable exertional angina and control subjects, * $p<0.05$ vs stable exertional angina and control subjects, ${ }^{\dagger \dagger} p<0.0001$ vs after treatment, ${ }^{\dagger} p<0.01$ vs after treatment, ${ }^{\dagger} p<0.05$ vs after treatment.

in left anterior descending artery, 1 in right coronary artery, and 1 in left circumflex artery). One of the 6 patients had intracoronary thrombolysis, after which rescue PTCA was performed. PTCA was successful in all patients (residual stenosis $\leq 50 \%$ ) and no abrupt closure of the infarct-related coronary artery occurred. In the remaining 4 patients with acute myocardial infarction, medical treatment was prescribed and coronary arteriography was performed 2-4 weeks after admission.

All the patients with unstable angina pectoris received medical treatment on admission: increasing doses or the addition of $\beta$-blockers in 4 patients, calcium antagonists in 
Table 4 Comparison of Platelet Aggregates Before and After $81 \mathrm{mg}$ of Aspirin Treatment in Patients With Stable Exertional Angina

\begin{tabular}{llll}
\hline \hline Platelet aggregates & Before aspirin & After aspirin & $p$ value \\
\hline Small-sized $(V)$ & $8.9 \pm 3.3 \times 10^{3}$ & $5.8 \pm 1.6 \times 10^{3}$ & $N S$ \\
Medium-sized $(V)$ & $1.4 \pm 0.3 \times 10^{3}$ & $9.5 \pm 1.6 \times 10^{2}$ & $N S$ \\
Large-sized $(V)$ & $2.7 \pm 0.3 \times 10^{3}$ & $1.7 \pm 0.4 \times 10^{3}$ & $<0.05$ \\
Optical density method $(\%)$ & $9.6 \pm 1.3$ & $5.9 \pm 0.8$ & $<0.05$ \\
\hline
\end{tabular}

Values are mean $\pm S E M$.

2 patients, long-acting nitrates in 6 patients, and aspirin in 12 patients. In all 13 patients with unstable angina pectoris, coronary arteriography was performed $2-4$ weeks after admission.

Twenty patients with acute coronary syndromes had significant organic stenosis of $90 \%$ or more of the diameter in the coronary arteries. Three patients with unstable angina pectoris and no organic coronary stenosis were documented to have had coronary spasms. All the patients with stable exertional angina had major coronary arterial stenosis of $\geq 90 \%$ of the diameter. The control subjects had no significant coronary stenosis and no coronary spasms $(\leq 25 \%$ diameter).

\section{Platelet Aggregation}

The number of small-sized platelet aggregates on admission was significantly higher in the acute coronary syndromes group than in the stable exertional angina group or the control group ( $p<0.0001$ ) (Fig 1 A; Table 3). Similarly for medium-sized $(\mathrm{p}<0.05)$, but not the large-sized platelet aggregates (Fig 1B,C; Table 3). Platelet aggregations on admission were significantly higher in the acute coronary syndromes group than in the stable exertional angina group or control group using the optical density method $(\mathrm{p}<0.01)$ (Fig 1D; Table 3).

Platelet aggregates were reexamined after medical treatment in the 23 patients with acute coronary syndromes. The number of small-sized platelet aggregates after treatment decreased significantly $(\mathrm{p}<0.0001)$, but was still significantly higher than that in the stable exertional angina group or control group $(\mathrm{p}<0.05)$ (Fig l A; Table 3 ). The number of medium-sized platelet aggregates after treatment also decreased significantly to the levels observed in the stable exertional angina group and control group $(\mathrm{p}<0.05)$ (Fig 1B; Table 3). However, the number of large-sized platelet aggregates after treatment did not change significantly (Fig 1C; Table 3). Platelet aggregations measured with the optical density method decreased significantly after treatment in the acute coronary syndromes group ( $\mathrm{p}<0.01)$ (Fig 1D; Table 3 ).

Twenty-two of the 23 patients in the acute coronary syndromes group were administrated $81 \mathrm{mg} /$ day of aspirin after hospitalization, as did 12 other patients with stable exertional angina as a preliminary study in which it was found that aspirin suppressed production of large-sized platelet aggregates, but had no influence on that of small or medium-sized platelet aggregates (Table4). These patients were not included in the present study. At baseline, the number of small-sized platelet aggregates was slightly higher in the patients with stable exertional angina in the preliminary study than in those of the present study, which may be due to the difference of the frequency of complicated coronary risk factors between the 2 groups, in particular the proportion of patients with the complication of diabetes mellitus were higher in the preliminary study ( 8 of 12 patients) than in the present study (5 of 14 patients).

\section{Discussion}

A platelet aggregability measurement system using a laser-light scattering method that can detect increases in the size of small-sized platelet aggregates, which can not be evaluated with conventional methods, has been developed 17,18 The new device has proved useful clinically through its ability to detect the enhanced responsiveness of platelets to low concentrations of physiological agents in various diseases ${ }^{17-19}$ The 2 phases of platelet aggregate formation (ie, the number of large-sized aggregates increases with a concomitant decrease in the number of small-sized platelet aggregates) represent the 'primary' and 'secondary' aggregation processes and so the production of small-sized platelet aggregates is very important for thrombus formation. With a high concentration, the number of large-sized platelet aggregates is too high to enable evaluation of different sized-platelet aggregates. In the present study we used a low concentration of ADP to investigate the most sensitive platelet aggregates, which may be why there were not many large-sized platelet aggregates.

Using the laser-light scattering method, we examined the platelet aggregability in patients with acute coronary syndromes, and the relationship between platelet aggregability on admission and after treatment. The number of small-sized platelet aggregates after treatment decreased significantly in patients with acute coronary syndromes, but was still significantly higher than the number in the stable exertional angina group and the control group. Coronary angioscopy shows that thrombus still exists in the infarct-related vessel within 1 month of acute myocardial infarction ${ }^{23}$ reflecting the persistence of a state of chronic hyperaggregability in patients with acute coronary syndromes after clinical stabilization. Using this laser-light scattering method, we recently reported that small-sized platelet aggregates increased after anginal attacks in patients with coronary spastic angina pectoris?

Mieke et al reported that spontaneous platelet aggregation in vitro was a useful biological marker for the prediction of coronary events and mortality in survivors with recent myocardial infarction.25 Recently, Goto et al reported that shear-induced platelet aggregation was enhanced in patients with acute myocardial infarction ${ }^{26}$ and previous studies reported that platelet aggregability increased in patients with diabetes mellitus ${ }^{27,28}$ This is one of the reasons for the slightly higher number of small-sized platelet aggregates in the patients with stable exertional angina in our preliminary study that examined the influence of aspirin. In the present study, there were no significant differences in the number of the patients with diabetes mellitus between the acute coronary syndromes group and the stable exertional angina group. We consider that the difference in the number of 
small-sized platelet aggregates reflected the pathological difference between these 2 groups.

In the present study, $81 \mathrm{mg} /$ day aspirin suppressed production of large-sized platelet aggregates, but did not influence the production of small- or medium-sized aggregates. Tohgi et al reported that, using ADP $1.0 \mu \mathrm{mol} / \mathrm{L}$ as an aggregating reagent, $330 \mathrm{mg} /$ day of aspirin suppressed production of large-sized platelet aggregates, but not that of small- or medium-sized platelet aggregates ${ }^{29}$ In general, aspirin can reduce cardiac mortality in patients with acute coronary syndromes ${ }^{22}$ and its mechanism may be not so much reducing the number of small-sized platelet aggregates, but rather reducing the process by which large-sized platelet aggregates form from small-sized ones. Furthermore, in platelet aggregation using ADP, thromboxane $\mathrm{A} 2$ does not influence small-sized platelet aggregates ${ }^{18}$ Roth et al demonstrated that exposure of platelets to aspirin inhibits cyclooxygenase and thus decreases production of thromboxane A2 30 Previous studies showed that ticlopidine's antiplatelet action is relatively specific against ADP aggregation, ${ }^{31}$ and Tohgi et al also reported that the number of small-sized platelet aggregates after $0.5 \mu \mathrm{mol} / \mathrm{L}$ ADP stimulation, and of medium and large-sized platelet aggregates after $1.0 \mu \mathrm{mol} / \mathrm{L} \mathrm{ADP}$ stimulation, was found to be lower in patients treated with $200 \mathrm{mg} /$ day of ticlopidine than in untreated patients. ${ }^{29}$ Therefore, we considered that ticlopidine influenced the production of small-sized platelet aggregates, and excluded the use of antiplatelet drugs except for aspirin. As it is thought that the decrease in the number of small-sized platelet aggregates after treatment reflects stabilization of condition of disease in patients with acute coronary syndromes, the present findings indicate that the formation of small-sized platelet aggregates may be a sensitive marker for platelet hyperaggregability.

\section{Acknowledgments}

This study was supported in part by a Research Grant for Cardiovascular Disease (10C-5 and 1/C-6) from the Ministry of Health and Welfare, and a grant-in-aid for scientific research (No.C11670692) from the Ministry of Education, Science, and Culture in Japan.

\section{References}

1. Fuster V, Badimon L, BadimonJJ, Chesebro JH: The pathogenesis of coronary artery disease and acute coronary syndromes. $N$ Engl J Med 1992; 326: 242-250

2. Libby P: Molecular bases of acute coronary syndromes. Circulation 1995; 91 : $2844-2850$

3. Falk E, Shah PK, Fuster V: Coronary plaque disruption. Circulation 1995; 92: 657-671

4. Oshima S, Ogawa H, Yasue H, Matsuyama K, Miyagi H: Increased plasma fibrinopeptide A levels during attacks induced by hyperventilation in patients with coronary vasospastic angina. $J$ Am Coll Cardiol 1989; 14: 150-154

5. Oshima S, Yasue H, Ogawa H, Okumura K, Matsuyama K: Fibrinopeptide $\mathrm{A}$ is released into the coronary circulation after coronary spasm. Circulation 1990; 82: 2222-2225

6. Ogawa H, Yasue H, Oshima S, Okumura K, Matsuyama K, Obata K: Circadian variation of plasma fibrinopeptide A level in patients with variant angina. Circulation 1989; 80: $1617-1626$

7. Eisenberg PR, Sherman LA, Schectman K, Perez J, Sobel BE, Jaffe AS: Fibrinopeptide A: A marker of coronary thrombosis. Circulation 1985; 71: $912-918$

8. Theroux P, Latour JG, Legar-Gauthier C, Lara JD: Fibrinopeptide A and platelet factor levels in unstable angina pectoris. Circulation 1987; 75: $156-162$

9. Sakamoto T, Yasue H, Ogawa H, Misumi I, Masuda T: Association of patency of the infarct-related coronary artery with plasma levels of plasminogen activator inhibitor activity in acute myocardial infarction. Am J Cardiol 1992; 70: 271 - 276

10. Masuda T, Yasue H, Ogawa H, Misumi I, Sakamoto T, Okubo H, et al: Plasma plasminogen activator inhibitor activity and tissue plasminogen activator levels in patients with unstable angina and those with coronary spastic angina. Am Heart $J$ 1992; 124: 314-319

11. Masuda T, Ogawa H, Miyao Y, Yu Q, Misumi I, Sakamoto T, et al: Circadian variation in fibrinolytic activity in patients with variant angina. Br Heart $J$ 1994; 71: 156-161

12. Weiberger I, Fuchs J, Davidson E, Rotenberg Z: Circulating aggregated platelets, number of platelet per aggregate, and platelet size during acute myocardial infarction. Am J Cardiol 1992; 70: 981 -983

13. Ault KA, Cannon CP, Mitchell J, McCahan J, Tracy RP, Novotny WF, et al: Platelet activation in patients after an acute coronary syndrome: Results from the TIMI-12 trial (Thrombolysis in Myocardial Infarction). J Am Coll Cardiol 1999; 33: 634-639

14. Ott I, Neumann F-J, Gawaz M, Schmitt M, Schomig A: Increased neutrophil-platelet adhesion in patients with unstable angina. Circulation 1996; 94: 1239-1246

15. Born GVR: Aggregation of blood platelets by adenosine diphosphate and its reversal. Nature 1962; 194: 927-929

16. Ries H, Braun G, Brehm G, Hiller E: Critical evaluation of platelet aggregation in whole human blood. Am J Clin Pathol 1986; 85: 50-56

17. Ozaki Y, Satoh K, Yatomi Y, Yamamoto T, Shirasawa Y, Kume S: Detection of platelet aggregates with a particle counting method using light scattering. Anal Biochem 1994; 218: 284-294

18. Satoh K, Ozaki Y, Qi R, Yang L, Asazuma N, Yatomi Y, et al: Factors that affect the size of platelet aggregates in epinephrineinduced activation: A study using the particle counting method based on light scattering. Thromb Res 1996; 81: 515-523

19. Eto K, Takeshita S, Ochiai M, Ozaki Y, Sato T, Isshiki T: Platelet aggregation in acute coronary syndromes: Use of a new aggregometer with laser scattering to assess platelet aggregability. Cardiovasc Res 1998; 40: $223-229$

20. Yasue H, Horio Y, Nakamura N, Fujii H, Imoto N, Sonoda R, et al: Induction of coronary artery spasm by acetylcholine in patients with variant angina: Possible role of the parasympathetic nervous system in the pathogenesis of coronary artery spasm. Circulation 1986; 74: 955-963

21. Geoffrey HT, Damian BAIS, Charles AC, John DR, Stefan NW, Ray $\mathrm{EG}$, et al: Concurrent morning increase in platelet aggregability and the risk of myocardial infarction and sudden cardiac death. $N$ Engl J Med 1987; 316: 1514-1518

22. Hennekenns CH, Dyken ML, Fuster V: Aspirin as a therapeutic agent in cardiovascular disease: A statement for healthcare professionals from the American Heart Association. Circulation 1997; 96: $2751-2753$

23. Belle EV, Lablanche JM, Bauters C, Renaud N, Mcfadden EP, Bertrand ME: Coronary angioscopic findings in the infarct-related vessel within 1 month of acute myocardial infarction. Circulation 1998; 97: 26-33

24. Miyamoto S, Ogawa H, Soejima H, Takazoe K, Sakamoto T, Yoshimura M, et al: Formation of platelet aggregates after attacks of coronary spastic angina pectoris. Am J Cardiol 2000; 85: 494-497

25. Mieke DT, Volkert MC, Frans JLC, Johan V: Platelet hyperreactivity and prognosis in survivors of myocardial infarction. $N$ Engl $J \mathrm{Med}$ 1990; 322: $1549-1554$

26. Goto S, Sakai H, Goto M, Ono M, Ikeda Y, Handa S, et al: Enhanced shear-induced platelet aggregation in acute myocardial infarction. Circulation 1999; 99: 608-613

27. Sakamoto $\mathrm{T}$, Ogawa $\mathrm{H}$, Kawano $\mathrm{H}$, Hirai N, Miyamoto S, Takazoe $\mathrm{K}$, et al: Rapid change of platelet aggregability in acute hyperglycemia: Detection by a novel laser-light scattering method. Thromb Haemost 2000; 83: 475-479

28. Fukuda K, Ozaki Y, Satoh K, Kume S, Tawata M, Onaya T, et al: Phosphorylation of myosin light chain in resting platelets from NIDDM patients is enhanced: Correlation with spontaneous aggregation. Diabetes 1997; 46: $488-493$

29. Tohgi H, Takahashi H, Watanabe K, Kuki H, Shirasawa Y: Development of large platelet aggregates from small aggregates as determined by laser-light scattering: Effect of aggregant concentration and antiplatelet medication. Thromb Haemost 1996; 75: 838-843

30. Roth GJ, Majerus PW: The mechanism of the effect of aspirin on human platelets: I. Acetylation of a particulate fraction protein. $J$ Clin Invest 1975; 56: 624-629

31. Saltiel E, Ward A, Ticlopidine: A review of its pharmacodynamic and pharmacokinetic properties, and therapeutic efficacy in plateletdependent disease states. Drugs 1987; 34: 222-262 